\title{
Differential thresholds for limb movement measured using adaptive techniques
}

\author{
L. A. JONES and I. W. HUNTER \\ McGill University, Montreal, Quebec, Canada \\ and \\ R. J. IRWIN \\ University of Auckland, Auckland, New Zealand
}

\begin{abstract}
Differential thresholds for limb movement were measured in 10 subjects, using the transformed up-down procedure. Subjects were required to indicate which of two random displacement perturbations delivered to their forearms had the larger standard deviation $(S D)$. The $S D$ of the reference signal was fixed for each experimental condition at one of seven values ranging from 0.05 to $3.2 \mathrm{~mm}$. The $S D$ of the other signal varied depending on the subject's response. Using this procedure, the differential threshold for limb movement was calculated to be $8 \%$, which is very similar to the thresholds estimated previously for changes in limb position (9\%) and force $(7 \%)$. The sensitivity of the human proprioceptive system to changes in limb displacement was much greater than anticipated, with subjects being able to resolve a 5- $\mu \mathrm{m}$ difference between two perturbations delivered to their arms.
\end{abstract}

The sensitivity of the human proprioceptive system to changes in joint angle or muscle force has usually been defined in terms of difference thresholds, which are measured by using some type of contralateral limb-matching procedure (Clark, Burgess, Chapin, \& Lipscomb, 1985; Gandevia \& McCloskey, 1977; Jones, 1989). The difference threshold divided by the reference stimulus intensity, known as Weber's fraction, is a useful index of sensory discrimination and has been used to compare the sensitivity of human observers to changes in force, limb position, and stiffness (Jones \& Hunter, 1990).

Many of these psychophysical studies have been devoted to the elucidation of the nature of the peripheral receptors that give rise to sensations of limb movement and position, by measuring the changes in sensory thresholds that occur following the temporary loss of input from one of these receptor populations (e.g., following anaesthesia of the skin or joints, or disengagement of the muscles). The results from these studies indicate that muscle receptors provide the principal source of proprioceptive sensations (Goodwin, McCloskey, \& Matthews, 1972; Matthews, 1988), but that input from joint and cutaneous receptors also contributes to our awareness of position and movement, particularly for the hand (Clark, Burgess, \& Chapin, 1986; Clark, Grigg, \& Chapin, 1989; Ferrell \& Smith, 1988).

This research was supported by the Medical Research Council of Canada and the Institute for Robotics and Intelligent Systems. The authors would like to acknowledge the assistance of Nat Durlach. I.W.H. is in the Department of Biomedical Engineering, McGill University; correspondence should be addressed to $\mathrm{L}$. A. Jones, School of Physical and Occupational Therapy, McGill University, 3654 Drummond St., Montreal, PQ, Canada H3G IY5.
Limb movement has generally been studied in terms of detection (i.e., absolute thresholds) rather than discrimination (i.e., difference thresholds), with the result that relatively little is known about the capacity of human subjects to discriminate between limb movements of varying amplitude or frequency. Studies of absolute thresholds have demonstrated that the ability to detect movements imposed on a joint depends on the velocity of the movement and the joint being moved. As the angular velocity of a movement increases, the absolute threshold for detecting the movement decreases (Ferrell, Gandevia, \& McCloskey, 1987; Gandevia, Hall, McCloskey, \& Potter, 1983; Goldscheider, 1889; Kokmen, Bossemeyer, \& Williams, 1977). When expressed in terms of angular rotations of the joint, these thresholds are smaller for more proximal joints, such as the shoulder and elbow, than for the more distal joints. However, if performance is defined in terms of linear displacements of the endpoint (i.e., the fingertip for the arm), the distal joints are superior to the proximal joints (Hall \& McCloskey, 1983). In these studies, subjects are required to indicate when they detect a movement and its direction. This condition generally results in higher thresholds than would be obtained if subjects only indicated the presence of a movement, because this appears to be detected prior to the movement's direction (Hall \& McCloskey, 1983).

Although a change in the position of a limb is usually experienced as a consequence of limb movement, it has been possible to dissociate these two aspects of proprioception experimentally by imposing extremely slow movements (i.e., $0.004^{\circ} / \mathrm{sec}$ ) on a joint. Using this procedure, Clark et al. (1985) have demonstrated that human subjects can make independent judgments of the static position and movement of a limb, and that the sense of 
limb position, unlike the sense of movement, is not influenced by the rate of joint rotation. These findings are supported by observations on the perceptual effects of muscle tendon vibration during which subjects can dissociate sensations of movement from those of position (McCloskey, 1973).

The sensitivity of human subjects to changes in limb position depends on the absolute position of the limb (Lloyd \& Caldwell, 1965), on whether the joint is proximal or distal (De Domenico \& McCloskey, 1987), on the spatial frame of reference adopted by the subject (Soechting, 1982; Worringham, Stelmach, \& Martin, 1987), and on the forces developed to maintain that position (Rymer \& D'Almeida, 1980; Worringham \& Stelmach, 1985). In addition, limb position is coded more accurately if the limb moves actively to a location rather than being passively positioned (Monster, Herman, \& Altand, 1973; Paillard \& Brouchon, 1968).

In most psychophysical studies of the proprioceptive system, researchers have used classical techniques such as the method of average error (Jones, 1989; Worringham et al., 1987) to determine sensory thresholds. Adaptive techniques (Levitt, 1971; Shelton, Picardi, \& Green, 1982) and signal detection analyses (Green \& Swets, 1989) have not been used as extensively as in other areas of perception, with some exceptions (e.g., Durlach et al., 1989; Kokmen et al., 1977; Pang, Tan, \& Durlach, 1991; Ross \& Brodie, 1987), in part because of problems associated with automating stimulus delivery and rapidly adjusting stimulus intensity. In previous studies, absolute thresholds for limb movement have been determined (Ferrell et al., 1987; Gandevia et al., 1983) and the differential threshold for limb position has been estimated (Erickson, 1974).

The objective of the present experiment was to apply criterion-free methods to determine differential thresholds for limb movement, using a two-alternative forced-choice method (2AFC) that involved a relatively fast adaptive procedure (Levitt, 1971). To our knowledge, this is the first use of Gaussian waveforms with the proprioceptive sensory modality, and once the effectiveness of the technique has been established, it will be possible to examine the influence of stimulus parameters such as bandwidth and probability density on the perception of limb movement.

\section{METHOD}

\section{Subjects}

Ten normal healthy adult subjects ( 5 women and 5 men) participated in the experiment. They had no known abnormalities of the neuromuscular system. They ranged in age from 25 to 54 years (mean: 35 years).

\section{Apparatus}

An experimental rig, measuring $1 \mathrm{~m}^{2}$ at the base and $2 \mathrm{~m}$ high, was constructed from solid square-bar aluminum struts $(40 \times 40 \mathrm{~mm}$ in section). Mounted in the center of the rig was a four-axis electrically adjustable seat, and in front of this chair was a horizontal beam on which aluminum elbow supports were bolted. A photograph of the apparatus is shown in Figure 1.

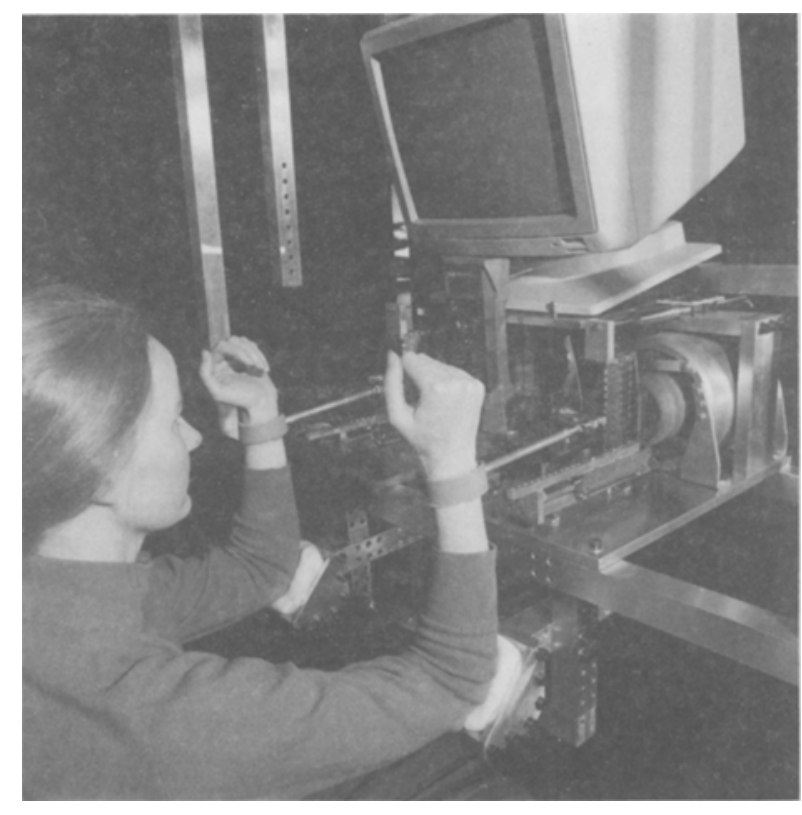

Figure 1. Photograph of a subject sitting in the apparatus used in the experiment. During the experiment, subjects were blindfolded and wore headphones.

Two electromagnetic linear motors (Sperry-Univac, 100-mm maximum linear displacement) were bolted to the rig and powered by 500-W power amplifiers (Techron Model 5530). The motors were servocontrolled by analog PID (proportional, integrative, and derivative) controllers with a high loop gain to achieve a stiffness of $10 \mathrm{kN} / \mathrm{m}$. Connected to the translation stage of each motor were an aluminum rod and cuff that were used with a velcro band to clamp the motor to the subject's wrist. An LVDT (linear variable differential transformer) displacement transducer (EMI Model SE 373, 100 -mm linear range) was mounted on each motor, with its movable core mechanically coupled to the translation stage. The displacement signals were passed through a signal conditioning module (modulator-demodulator) prior to being filtered by a second-order Butterworth filter ( $300 \mathrm{~Hz}$ low-pass).

Strain-gauge (full-bridge, temperature-compensated) force transducers were mounted on the translation stage of the motor. The force signals were amplified (140 dB CMRR) prior to being recorded. These signals were used to monitor whether there was any change in the voluntary forces produced by the subjects (and hence the stiffness of the limb) during the experiment.

The motors delivered a randomly varying displacement to each wrist simultaneously for $6 \mathrm{sec}$. The displacement signals, shown in Figure 2, were generated according to the following procedure: $5 \mathrm{sec}$ of Gaussian white noise (mean of 0 ) were generated numerically and then convolved by a \pm 0.5 -sec length, symmetric, twosided ( 0 phase) digital low-pass (cutoff $15 \mathrm{~Hz}$ ) filter. This resulted in a 6-sec signal conveniently tapered for 0.5 -sec at either end as illustrated in Figure 2. The standard deviation $(S D)$ of the Gaussian white noise was specified as either the reference $S D\left(S D_{\text {ref }}\right)$ or the signal $S D\left(S D_{\text {sig }}\right)$. In this experiment, $S D$ is equivalent to the rms amplitude. The power spectrum of the displacement perturbations is shown in Figure 3 . The maximum displacement delivered to the arms was limited to $\pm 10 \mathrm{~mm}$. The random displacement signals delivered by the two motors were the same except for their $S D$ s and that one was a time-reversed version of the other.

Force and displacement signals were recorded during the displacement perturbations via 12-bit A/Ds (with digitally programmable gains) at $100 \mathrm{~Hz}$, and the motors were controlled via 16-bit D/As 
clocked at $100 \mathrm{~Hz}$. The subject's response on each trial was recorded. The $A / D$ s and D/As formed part of a single board I/O system (Burr Brown Model PCI-602W) residing on an IBM R6000/320 computer. This computer was used for experimental control, data acquisition, digital servocontrol, and subsequent data analysis. Matlab (Math Works Inc., South Natick, MA), an interactive software package for scientific and numeric computation, was used to generate the signals. The experimental procedures and servocontrols were all written in the $C$ programming language.

The digital I/O on the computer was used to control a function generator that produced a $750-\mathrm{Hz}$ tone, via headphones, during the displacements. This auditory signal served to obscure any auditory cues arising from the motors and indicated to the subject the observation interval. This was particularly important for the smallest amplitude movements, whose onset and termination were often difficult to detect.

Differences in the inertias of the subjects' forearms and trial-totrial variation in the stiffness of the subjects' elbow joints (due to muscle contraction or co-contraction of the biceps and triceps muscles) resulted in a discrepancy between the programmed $S D_{\text {ref }}$ and the measured $S D_{\text {ref }}$ (and also $S D_{\text {sig }}$ and the measured $S D_{\text {sig }}$ ). A digital servocontrol algorithm was therefore implemented to make the measured value as close as possible to the desired value. The procedure multiplied $S D_{\text {ref }}$ (measured on the current trial) by a correction factor associated with each motor. A similar procedure was implemented for $S D_{\text {sig. }}$. In order to maximize utilization of the available dynamic range of the $A / D$ s the gain of both $A / D$ s was increased by a factor of 10 when $S D_{\text {ref }}$ was less than $1 \mathrm{~mm}$.

\section{Procedure}

The subjects sat in the chair with each elbow joint resting in a molded plastic cast (Aquaplast) that was in turn encased in an elbow support. The position of these supports was adjusted until the angle between the upper arm and forearm was $90^{\circ}$. During the experiment, the subjects were blindfolded to prevent them from using visual cues to facilitate the perception of movement, and they wore headphones delivering a $750-\mathrm{Hz}$ tone that masked any auditory cues arising from the motors.

A single-interval forced-choice paradigm was used, with trialby-trial feedback giving the correct answer. On each trial, a ran-

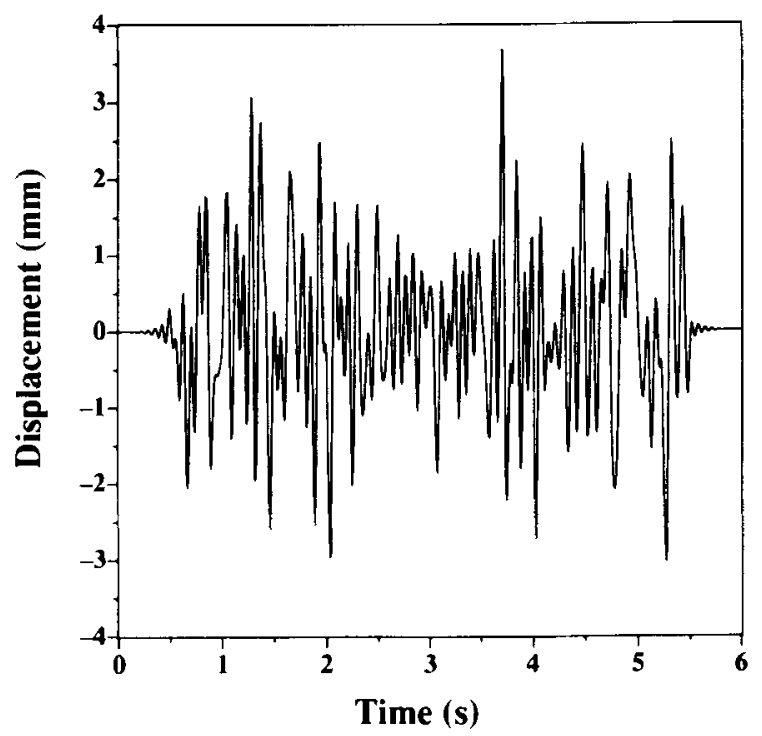

Figure 2. A Gaussian displacement perturbation with a mean of zero and a standard deviation $(S D)$ of $1 \mathrm{~mm}$. This signal was multiplied by the required $S D$ to achieve the actual signal used in any trial.

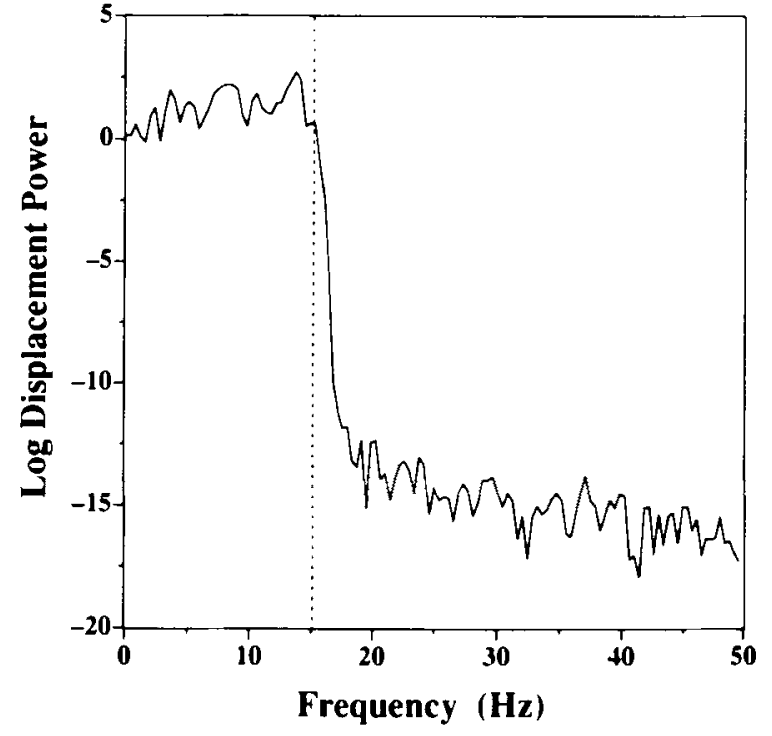

Figure 3. Power spectrum of the displacement perturbation. The dashed line indicates the low-pass filter cutoff at $15 \mathrm{~Hz}$.

dom displacement perturbation was delivered simultaneously to each arm for $6 \mathrm{sec}$. The subjects were instructed to relax their arms during the perturbation. At the end of the perturbation, they were required to indicate within $20 \mathrm{sec}$ which displacement had the larger standard deviation by pulling the appropriate arm towards them (a force of $10 \mathrm{~N}$ had to be generated for the response to be recorded). After a 0.5 -sec pause, the correct response (i.e., the arm that had experienced the larger amplitude displacement) was indicated to the subject by a $2-\mathrm{mm} 100$-msec pulse delivered to the correct arm.

$S D_{\text {ref }}$ was fixed for each block of 50 trials (experimental condition) at one of seven values: $0.05,0.1,0.2,0.4,0.8,1.6$, and $3.2 \mathrm{~mm}$. The order in which these seven conditions was presented was randomized across subjects. There was a rest period of 3-5 min between each block of 50 trials, during which the subject's arms were released from the apparatus. At the start of each experimental condition, $S D_{\text {sig }}$ was set to twice that of $S D_{\text {ref; }}$ thereafter, the difference between $S D_{\text {ret }}$ and $S D_{\text {sis }}$ decreased or increased, depending on the subject's responses. A transformed up-down procedure was used (Levitt, 1971) to track the subject's threshold, with the absolute difference between $S D_{\text {ref }}$ and $S D_{\text {siz }}$ being halved after two successive correct responses and doubled after one incorrect response. These rules seek a stimulus level that corresponds to $71 \%$ correct performance. This procedure was selected on the basis of extensive numerical simulations, and the algorithm chosen was based on a tradeoff between converging too quickly to a value not close to the actual threshold and converging too slowly and therefore requiring long experimental durations. Because it was the difference between $S D_{\text {ref }}$ and $S D_{\text {sig }}$ that was relevant, it was randomly determined on each trial whether the difference was added to or subtracted from $S D_{\text {ref }}$ to create $S D_{\text {sig. }}$. In order to control for the space error-that is, errors in measurement that can arise when stimuli are presented to different receptive areas, such as the left and right arms (Gescheider, 1985) $-S D_{\text {ref }}$ and $S D_{\text {sig }}$ were randomly assigned to the left and right motor on each trial.

\section{RESULTS}

The high stiffness of the motors, coupled with the implementation of digital servocontrol algorithms, resulted in the difference between the measured and programmed 
$S D_{\text {ref }}$ values being less than $2 \%$ by the third stimulus presentation. This indicates that any voluntary movements made by subjects were compensated for by the servocontrol systems.

The subjects were not given any explicit instructions regarding the cues to use to determine the relative variation of the two displacements, and they did not receive any practice trials. Nevertheless, they did not report any difficulties in performing the task. They typically made responses within $5 \mathrm{sec}$ of the termination of the displacement perturbations. They did comment, however, on difficulties in perceiving the onset and termination of the smallest amplitude signal (i.e., $S D_{\text {ref }}$ of $0.05 \mathrm{~mm}$ ) and indicated that they used the onset and offset of the tone for this purpose.

The decrease in the amplitude of the absolute difference between $S D_{\text {ref }}$ and $S D_{\text {sig }}$ during a typical experimental run is shown in Figure 4. As can be seen in the figure, after approximately 15-20 trials, the subject's responses were hovering around the threshold level, defined as the level at which $71 \%$ correct performance was attainable. The differential threshold was defined as the geometric mean of the absolute difference between the measured $S D_{\text {ref }}$ and the measured $S D_{\text {sig }}$ for the last 30 trials and was calculated for each subject at each displacement amplitude.

The mean differential thresholds estimated from the group data are shown in Figure 5, together with the relation between the differential threshold and stimulus intensity predicted by Weber's law. These data are plotted logarithmically, because of the geometric spacing of the reference signal amplitudes. The thresholds increase monotonically and essentially linearly with the amplitude of the reference stimulus. The data illustrated in Figure 5 reveal that, at the smallest amplitude $\left(S D_{\text {ref }}=0.05 \mathrm{~mm}\right)$,

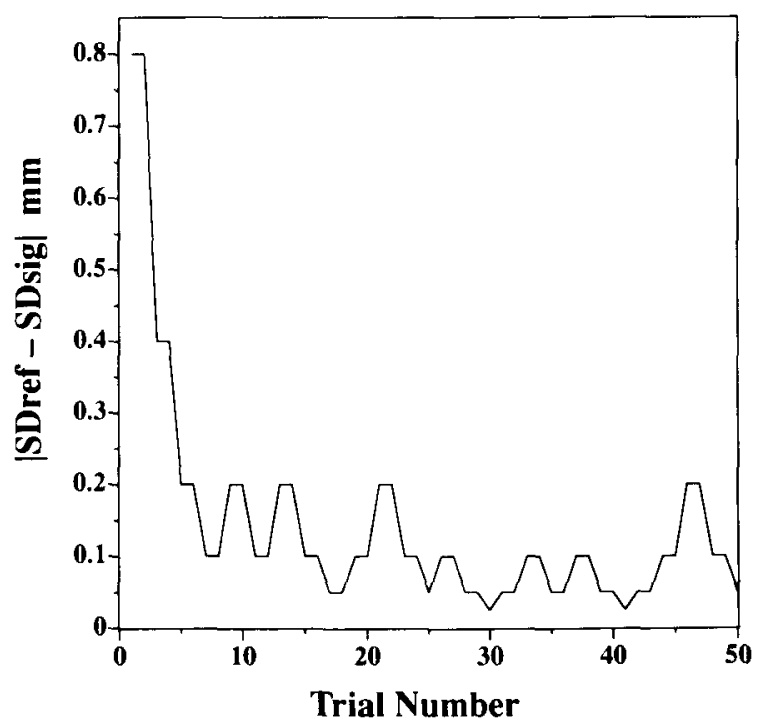

Figure 4. The absolute difference between $S D_{\text {ref }}$ and $S D_{\text {sg }}$ during a typical experimental run. $S D_{\text {ref }}$ is set at $0.8 \mathrm{~mm}$ in this example.

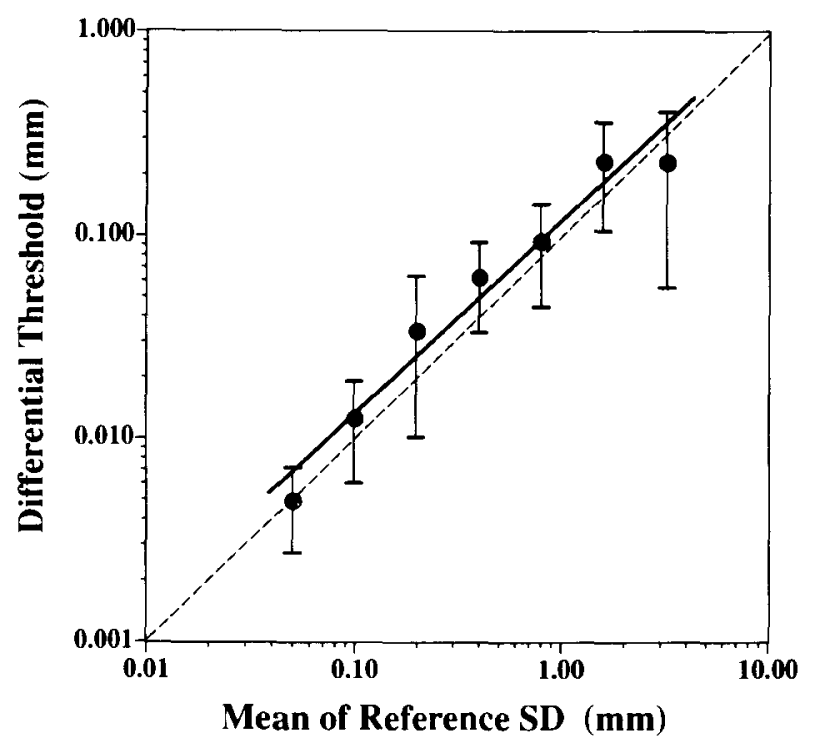

Figure 5. Mean differential thresholds estimated from the data from 10 subjects. The bars represent one standard deviation on either side of the mean. The dashed line with a unit slope represents the relationship between the differential threshold and stimulus intensity predicted by Weber's law. The solid line is the least squares regression line fitted to the data on logarithmic coordinates (i.e., a power function).

the subjects were able to distinguish a $0.005-\mathrm{mm}$ (i.e., $5-\mu \mathrm{m})$ difference between the two signals. The linear regression line fitted to these data (i.e., the linear values) accounted for $83 \%$ of the variance, and the slope of this line, which is the Weber fraction for limb movement, is 0.076 . The intercept is 0.0258 with a $95 \%$ confidence interval of $0.0258 \pm 0.0578$. Since zero is within the confidence interval of the intercept, these data are in accord with the original version of Weber's law, which does not involve an added constant (see Laming, 1986). For the individual data, the Weber fractions ranged from 0.04 to 0.19 . A comparison between the Weber fractions obtained from this small group of female and male subjects revealed that there was a significant difference between the sexes, with men obtaining lower thresholds than women $[M=$ 0.058 and 0.092 , respectively; $t(8)=4.25, p<.01$ ].

Force was measured from each arm during the experiment in order to see whether there were any systematic variations associated with different displacement amplitudes. It was hypothesized that subjects might increase the stiffness of the elbow joint during the very small displacements in order to facilitate perception of the limbs' movements. Analysis of the mean variation (i.e., $S D$ ) in force during the displacements indicated that the forces produced by subjects were very small, as is illustrated in Table 1 where the mean variation in the forces recorded from the left and right arms during the smallest, median, and largest displacement perturbations are shown.

A two-way analysis of variance of the forces shown in Table 1 with arm and reference signal amplitude as factors revealed a main effect of amplitude $[F(2,54)=$ 
Table 1

Mean Standard Deviation (SD) in Force (in N) Recorded From the Left and Right Arms During Three Experimental Conditions

\begin{tabular}{|c|c|c|c|c|c|c|}
\hline \multirow[b]{3}{*}{ Subject } & \multicolumn{6}{|c|}{ Reference Signal $S D(\mathrm{~mm})$} \\
\hline & \multicolumn{2}{|c|}{0.05} & \multicolumn{2}{|c|}{0.4} & \multicolumn{2}{|c|}{3.2} \\
\hline & Left & Right & Left & Right & Left & Right \\
\hline 1 & 0.46 & 0.21 & 0.71 & 1.06 & 3.75 & 8.10 \\
\hline 2 & 0.74 & 0.73 & 0.41 & 1.07 & 3.30 & 2.54 \\
\hline 3 & 1.01 & 1.47 & 1.46 & 1.93 & 4.35 & 6.64 \\
\hline 4 & 0.42 & 0.64 & 0.67 & 0.84 & 4.68 & 6.34 \\
\hline 5 & 0.31 & 0.23 & 0.49 & 0.43 & 4.03 & 2.25 \\
\hline 6 & 1.04 & 2.00 & 1.04 & 1.45 & 3.99 & 3.34 \\
\hline 7 & 0.26 & 0.32 & 0.47 & 0.77 & 5.09 & 2.21 \\
\hline 8 & 0.32 & 0.31 & 0.50 & 0.80 & 4.09 & 2.52 \\
\hline 9 & 0.28 & 0.25 & 0.62 & 0.69 & 4.48 & 5.41 \\
\hline 10 & 0.44 & 0.52 & 0.55 & 0.79 & 4.05 & 3.72 \\
\hline
\end{tabular}

$86.66, p<.001]$, but not of arm $[F(1,2)=18.51$, $p>.05]$, and no significant interaction between arm and amplitude $[F(2,54)=0.04, p>.05]$. Post hoc analysis (Tukey test) of the main effect of reference signal amplitude revealed that the forces produced during the largest perturbation $(3.2 \mathrm{~mm})$ were significantly greater $(p<.05)$ than those produced during the other two perturbations $(0.4$ and $0.05 \mathrm{~mm})$.

\section{DISCUSSION}

The results from this experiment indicate that subjects can reliably detect an $8 \%$ difference in the amplitude of random displacements delivered to their forearms over a stimulus range of $64: 1$ and that the perceptual resolution of the forearm appears to be much greater than reported previously (Hall \& McCloskey, 1983). Using quite a different experimental procedure, Durlach et al. (1989) reported that the just noticeable difference in length that could be discriminated by the hand was $1 \mathrm{~mm}$ for reference lengths in the range of $10-20 \mathrm{~mm}$. The task in their experiment involved perceiving a change in the static position (i.e., finger span) of the fingers, which is very different from the present situation, and the apparatus that they used was limited to a minimum length of $10 \mathrm{~mm}$.

The Weber fraction is a useful index of sensory discrimination that can be used to compare the relative sensitivities of different sensory modalities and of different aspects of the same sensory system. Comparison of the Weber fractions estimated for the three components of the proprioceptive system-namely, the perception of limb movement, position (Jones \& Hunter, 1990, from data in Erickson 1974), and force (Jones, 1989)-reveal a remarkable consistency in the values obtained $(0.08,0.09$, and 0.07 , respectively). The similarity in these values is surprising, given the considerable variation in the procedures used to determine them and in the sources of afferent information upon which the judgments are based. In this context, it is interesting that the Weber fraction for elastic stiffness (i.e., force/displacement) is $\mathbf{0 . 2 3}$ (Jones
\& Hunter, 1990), which indicates that subjects are much less consistent in discriminating changes in stiffness as opposed to force and movement.

The perception of limb movement depends primarily on sensory signals originating in the muscle spindle receptors (Clark et al., 1985; Goodwin et al., 1972). The relative contribution of joint and cutaneous receptors to proprioception is still subject to debate (see reviews by Clark \& Horch, 1986; Matthews, 1988), although recent studies suggest that cutaneous mechanoreceptors play a facilitatory role, in that loss of cutaneous input results in increased thresholds for detecting movements of the fingers, even when skin surfaces not involved in the movement are anesthetized (Clark et al., 1986; Clark et al., 1985).

In the present investigation, subjects probably relied on both proprioceptive and cutaneous signals to determine the relative variation of the two displacement signals. The remarkable resolution obtained at extremely small displacement amplitudes (i.e., discriminating a difference of $5 \mu \mathrm{m}$ in a reference signal of $0.05 \mathrm{~mm}$ ) probably reflects the perception of skin indentation - that is, vibrotactile stimulation-rather than movement of the forearm, which in this situation would have averaged less than $0.02^{\circ}$. Hall and McCloskey (1983) reported that the absolute threshold for detecting movements through the midrange of elbow joint excursion (i.e., around a joint angle of $90^{\circ}$ ) is approximately $0.08^{\circ}$, and that this remains constant with movement velocities between $1.25^{\circ}$ and $80^{\circ} / \mathrm{sec}$ (the peak velocities experienced by subjects in the present study were around $360 \% / \mathrm{sec}$ ). These absolute thresholds were calculated from single unidirectional movements imposed on the forearm, and it is possible that the difference threshold for movement could be much less (negative masking), as has been shown for the detection and discrimination of vibrotactile stimuli. Craig (1974) reported that the absolute threshold for detecting a $160-\mathrm{Hz}$ sinusoidal vibration applied to the finger tip is $0.1 \mu \mathrm{m}$, but that subjects can discriminate a difference as small as $0.04 \mu \mathrm{m}$ at a standard amplitude of $0.1 \mu \mathrm{m}$.

The sensitivity of human subjects to changes in the intensity of vibrotactile stimuli varies as a function of the size of the stimulated area, the frequency of vibration, the body site tested, skin temperature, and the presence of a static surround (see review by Sherrick \& Cholewiak, 1986). In particular, the absolute threshold for detecting sinusoidal vibratory movements of a small probe on the skin is highly dependent on the frequency of the stimulus. For the thenar eminence of the hand, the peak-topeak amplitude of the absolute threshold is typically less than $1 \mu \mathrm{m}$ at frequencies between 100 and $300 \mathrm{~Hz}$, whereas it is $70 \mu \mathrm{m}$ at $5 \mathrm{~Hz}$ (Mountcastle, LaMotte, \& Carli, 1972). For the hairy skin of the forearm, the pattern of results is similar but the thresholds are higher, with the minimum absolute threshold at $100 \mathrm{~Hz}$ being approximately $6 \mu \mathrm{m}$ (Merzenich \& Harrington, 1969). These studies have all focused on the measurement of absolute thresholds with the use of sinusoidal stimuli, and there 
do not appear to be any data available with regard to the detection of Gaussian noise waveforms.

In the present experiment, the area of contact around the wrist was typically $0.01 \mathrm{~m}^{2}$, and frequencies up to $15 \mathrm{~Hz}$ were delivered. The differential threshold for vibrotactile intensity is usually around $20 \%-25 \%$ (Craig, 1974 ), and it is $17 \%$ for pressure applied to the skin of the forearm (Kiesow, and Gatti \& Dodge, cited in Laming, 1986), both of which are considerably higher than the threshold measured here. It seems unlikely, therefore, that cutaneous cues were the sole source of information used by subjects to discriminate between the perturbations applied to their arms.

Comparisons between the findings from the present investigation and other studies must be made cautiously, however, in that fixed (usually sinusoidal) stimuli have typically been used in earlier work. One could argue that the findings described here are in fact "stimulus limited" - that is, the Weber fractions reported are a result of using Gaussian noise waveforms. At present, it is not known what effect the stimulus waveform has on the Weber fraction for limb movement, but from considerations of the physiology of the proprioceptive system (Matthews, 1988), it seems probable that the Weber fraction would be similar for other classes of stimuli such as sinusoidal or rectangular waveforms. It also seems likely that the Weber fraction would change for signals of different bandwidths. These are empirical questions that await further investigation.

It had been anticipated that the Weber fraction for limb movement would increase as the reference signal became very small, because this is a common feature of many sensory modalities and reflects the operation of sensory systems near absolute threshold levels (Gescheider, 1985). The lack of any change in the Weber fraction at low stimulus intensities and the relative invariance of Weber fractions at higher amplitudes (see Figure 5) do, however, accord with Laming's (1986) analysis of Weber's law, which he maintains is valid right down to the absolute threshold for difference discrimination (i.e., discriminating between two separate stimuli of magnitudes $X$ and $X+\Delta X$ ), as opposed to increment detection (i.e., detecting a single increment added to a continuous background stimulus of level $X$ ), where the departure from Weber's law at low levels is usually seen. In the present study, subjects were clearly required to discriminate between two distinct stimuli, and in this task, the data are in accord with the original version of Weber's law (see Figure 5). It is interesting that Weber's law also holds for discriminating the level (i.e., rms amplitude) of Gaussian noise but not for the amplitude of a pure tone (Laming, 1986). The absence of any change in the Weber fraction is also interesting in that it suggests a continuity in perceptual processes with the relative contributions of the cutaneous and proprioceptive systems changing as the stimulus amplitude increases.

Discontinuities in psychophysical functions for absolute sensitivity have been described previously for transitions between the operation of one type of sensory receptor and another. For example, Verrillo (1963) found that the absolute threshold for detecting vibration is independent of stimulus frequency at low frequencies and is a U-shaped function of frequency at higher frequencies, and this break in the psychophysical function appeared to be related to the operation of two classes of cutaneous mechanoreceptor. Similarly, the recovery of sensitivity after exposure of the eye to intense light, which is known as the dark adaptation curve, is a biphasic process, with a relatively rapid reduction in the absolute threshold during the first phase and then a more gradual decrease in threshold. The biphasic curve is caused by the differing rates of recovery of the rods and cones in the retina from the intense stimulation, with the first phase reflecting the operation of the cones and the latter part the rods (Gescheider, 1985). Similar changes in psychophysical functions have not been reported for difference thresholds, and there is no reason to assume that these functions should in fact be similar. The lack of any marked change in the psychophysical function described in this experiment may be due to the fact that both receptor systems contribute to the perception of limb movement under normal conditions, and that there is not an abrupt change in the relative contributions of the two types of sensory input.

In summary, the differential threshold for limb movement was calculated with the use of the transformed updown procedure and was found to be $8 \%$, which is very similar in magnitude to the Weber fraction calculated for limb position and force. The sensitivity of the proprioceptive system to changes in limb displacement was much smaller than anticipated, with subjects being able to resolve a $5-\mu \mathrm{m}$ difference between two perturbations delivered to their arms. It remains to be tested whether these thresholds will change as a function of the frequency content of the displacement signals presented to subjects.

\section{REFERENCES}

Clark, F. J., Burgess, R. C., Chapin, J. W. (1986). Proprioception with the proximal interphaleangeal joint of the index finger. Brain, 109, 1195-1208.

Clark, F. J., Burgess, R. C., Chapin, J. W., Lipscomb, W. T. (1985). Role of intramuscular receptors in the awareness of limb position. Journal of Neurophysiology, 54, 1529-1540.

Clark, F. J., Grigg, P., Chapin, J. W. (1989). The contribution of articular receptors to proprioception with the fingers in humans. Journal of Neurophysiology, 61, 186-193.

Clark, F. J., \& Horch, K. W. (1986). Kinesthesia. In K. Boff, L. Kaufman, \& J. P. Thomas (Eds.), Handbook of perception and human performance (Vol. 1, pp. 13-1-13-62). New York: Wiley.

CraIG, J. C., (1974). Vibrotactile difference thresholds for intensity and the effect of a masking stimulus. Perception \& Psychophysics, 15, 123-127.

De Domenico, G., \& McCloskey, D. I. (1987). Accuracy of voluntary movements at the thumb and elbow joints. Experimental Brain Research, 65, 471-478.

Durlach, N. I., Delhorne, L. A., Wong, A., Ko, W. Y., RABinowitz, W. M., \& Hollerbach, J. (1989). Manual discrimination and identification of length by the finger-span method. Perception \& Psychophysics, 46, 29-38.

Erickson, R. P. (1974). Parallel "population" neural coding in feature extraction. In F. O. Schmitt \& F. G. Worden (Eds.), The neurosciences: Third Study Program (pp. 155-169). Cambridge, MA: MIT Press. 
Ferrell, W. R., Gandevia, S. C., McCloskey, D. I. (1987). The role of joint receptors in human kinaesthesia when intramuscular receptors cannot contribute. Journal of Physiology, 386, 63-71.

Ferrell, W. R., Smith, A. (1988). Position sense at the proximal interphalangeal joint of the human index finger. Journal of Physiology, 399, 49-61.

Gandevia, S. C., Hall, L. A., McCloskey, D. I., \& Potter, E. K. (1983). Proprioceptive sensation at the terminal joint of the middle finger. Journal of Physiology, 335, 507-517.

Gandevin, S. C., MCCloskey, D. I. (1977). Changes in motor commands as shown by changes in perceived heaviness, during partial curarization and peripheral anaesthesia in man. Joumal of Physiology, 272, 673-689.

Gescheider, G. A. (1985). Psychophysics: Method, theory, and application (2nd ed.). Hillsdale, NJ: Erlbaum.

GolDSCHEIDER, A. (1889). Untersuchungen über den Muskelsinn. Archiv für Anatomie und Physiologie, 3, 369-502.

Goodwin, G. M., McCloskey, D. I., Matthews, P. B. C. (1972). The contribution of muscle afferents to kinaesthesia shown by vibration induced illusions of movement and by the effects of paralysing joint afferents. Brain, 95, 705-748.

GREEN, D. M., SWETS, J. A. (1989). Signal detection theory and psychophysics. Los Altos, CA: Peninsula.

HALL, L. A., \& MCCLOSKEY, D. I. (1983). Detections of movements imposed on finger, elbow and shoulder joints. Joumal of Physiology, $335,519-533$.

JoNes, L. A. (1989). Matching forces: Constant errors and differential thresholds. Perception, 18, 681-687.

Jones, L. A., Hunter, I. W. (1990). A perceptual analysis of stiffness. Experimental Brain Research, 79, 150-156.

Kokmen, E., Bossemeyer, R. W., \& Williams, W. J. (1977). Quantitation of motion perception in the digits: A psychophysical study in normal human subjects. Annals of Neurology, 2, 279-284.

Laming, D. (1986). Sensory analysis. London: Academic Press.

LEVITT, H. (1971). Transformed up-down methods in psychoacoustics. Journal of the Acoustical Society of America, 49, 467-477.

LLOYD, A. J., \& CALDWELL, L. S. (1965). Accuracy of active and passive positioning of the leg on the basis of kinesthetic cues. Joumal of Comparative \& Physiological Psychology, 60, 102-106.

MAtтhews, P. B. C. (1988). Proprioceptors and their contribution to somatosensory mapping: Complex messages require complex processing. Canadian Joumal of Physiology \& Pharmacology, 66, 430-438.

MCCloskey, D. I. (1973). Differences between the senses of movement and position shown by the effects of loading and vibration of muscles in man. Brain Research, 63, 119-131.

Merzenich, M. M., HARRington, T. (1969). The sense of flutter- vibration evoked by stimulation on the hairy skin of primates: Comparison of human sensory capacity with the responses of mechanoreceptive afferents innervating the hairy skin of monkeys. Experimental Brain Research, 9, 236-260.

Monster, A. W., Herman, R., Altand, N. R. (1973). Effect of the peripheral and central "sensory" component in the calibration of position. In J. E. Desmedt (Ed.), New developments in electromyography and clinical neurophysiology (Vol. 3, pp. 383-403). Basel: Karger.

Mountcastle, V. B., LaMotte, R. H., \& CarL, G. (1972). Detection thresholds for stimuli in humans and monkeys: Comparison with threshold events in mechanoreceptive afferent nerve fibers inservating the monkey hand. Joumal of Neurophysiology, 35, 122-136.

Paillard, J., Brouchon, M. (1968). Active and passive movements in the calibration of position sense. In S. J. Freedman (Ed.), The neuropsychology of spatially oriented behavior (pp. 37-55). Homewood, IL: Dorsey.

PANG, X. D., TAN, H. Z., DuRlaCh, N. I. (1991). Manual discrimination of force using active finger motion. Perception \& Psychophysics, 49, 531-540.

Ross, H. E., Brodie, E. E. (1987). Weber fractions for weight and mass as a function of stimulus intensity. Quarterly Joumal of Experimental Psychology, 39A, 77-88.

Rymer, W. Z., D'AlmeidA, A. (1980). Joint position sense: The effects of muscle contraction. Brain, 103, 1-22.

Shelton, B. R., Picardi, M. C., Green, D. M. (1982). Comparison of three adaptive psychophysical procedures. Joumal of the Acoustical Sociery of America, 71, 1527-1533.

Sherrick, C. E., Cholewiak, R. W. (1986). Cutaneous sensitivity. In K. Boff, L. Kaufman, \& J. P. Thomas (Eds.), Handbook of perception and human performance (Vol. 1, 12-1-12-58). New York: Wiley.

Soechting, J. F. (1982). Does position sense at the elbow reflect a sense of elbow joint angle or one of limb orientation? Brain Research, 248, 392-395.

VERRILLO, R. T. (1963). Effect of contactor area on the vibrotactile threshold. Joumal of the Acoustical Society of America, 35, 1962-1966.

Worringham, C. J., Stelmach, G. E. (1985). The contribution of gravitational torques to limb position sense. Experimental Brain Research, 61, 38-42.

Worringham, C. J., Stelmach, G. E., a Martin, Z. E. (1987). Limb segment inclination sense in proprioception. Experimental Brain Research, 66, 653-658.

(Manuscript received October 18, 1991; revision accepted for publication April 29, 1992.) 